\title{
An exact solution of a quasilinear Fisher equation in cylindrical coordinates
}

\author{
Ashfaque H. Bokhari, M. T. Mustafa and F. D. Zaman \\ Department of Mathematics and Statistics, King Fahd University \\ of Petroleum and Minerals, Dhahran 31261, Saudi Arabia \\ abokhari@kfupm.edu.sa, tmustafa@kfupm.edu.sa, fzaman@kfupm.edu.sa
}

\begin{abstract}
Lie symmetry method is applied to analyze Fisher equation in cylindrical coordinates. Symmetry algebra is found and symmetry invariance is used to reduce the equation to a first order ODE. The first order ODE is further analyzed to obtain exact solution of Fisher equation in explicit form.
\end{abstract}

Keywords: partial differential equations, Fisher equation, exact solutions.

The Lie symmetry approach to study a non-linear partial differential equation is based on finding its symmetry algebra which leads to reductions and construction of exact solutions of the PDE under investigation. Symmetry solutions of Fisher equation [6]

$$
\frac{\partial u}{\partial t}-\frac{\partial^{2} u}{\partial x^{2}}=u(1-u)
$$

which arises in Heat Transfer, Biology \& Ecology, have been investigated in $[1,3,5]$. It is well known for heat equation models that, in general, the thermal properties of the medium are not constant and may depend upon the temperature [7]. This leads to the following generalization

$$
\frac{\partial u}{\partial t}-\frac{\partial}{\partial x}\left(u \frac{\partial u}{\partial x}\right)=u(1-u)
$$

If $\underline{\nabla}$ denotes the gradient operator then, in higher dimensions, this equation takes the form

$$
\frac{\partial u}{\partial t}-\underline{\nabla} \cdot\left(u \frac{\nabla u}{\partial x}\right)=u(1-u),
$$

where complete symmetry analysis of this equation needs to be carried out. Here, we investigate Equation (2) in case of cylindrical domains with $u$ depending only on radius. If the radial variable is denoted by $x$ then the corresponding 
Fisher equation is given by

$$
\frac{\partial u}{\partial t}-\frac{1}{x} \frac{\partial}{\partial x}\left(x u \frac{\partial u}{\partial x}\right)=u(1-u) .
$$

Our aim is to apply classical Lie symmetry method to find an exact solution of Equation (3). Firstly the symmetries of Equation (3) are found and reduction to second order ODE is obtained through a suitable symmetry invariance. The reduced ODE is further analyzed to obtain exact solution of Equation (3) in explicit form.

We use the standard method, cf. [2, 4], for finding the infinitesimal symmetry generators of Equation (3). To obtain the symmetries, we take the infinitesimal generator of a point symmetry of the form

$$
X=\xi(x, t, u) \frac{\partial}{\partial x}+\tau(x, t, u) \frac{\partial}{\partial t}+\phi(x, t, u) \frac{\partial}{\partial u} .
$$

Let $X^{2}$ denote the second prolongation of the vector field $X$. Using the invariance condition $\left.X^{2}(E)\right|_{E=0}=0$ gives the following over-determined system of determining equations.

$$
\begin{aligned}
& \xi_{u}=0=\tau_{u}=\tau_{x} \\
& -u \xi+x \phi+x^{2} \xi_{t}-u x \xi_{x}+u x \tau_{t}+2 x^{2} \phi_{x}-u x^{2} \xi_{x x}+2 u x^{2} \phi_{x u}=0 \\
& -x \phi+2 u x \phi-u x \tau_{t}+u^{2} x \tau_{t}+x \phi_{t}+u x \phi_{u}-u^{2} x \phi_{u}-u \phi_{x}-u x \phi_{x x}=0 \\
& \phi-2 u \xi_{x}+u \tau_{t}=0 \\
& -2 \xi_{x}+\tau_{t}+\phi_{u}+u \phi_{u u}=0 .
\end{aligned}
$$

The solution to above system gives

$$
\begin{gathered}
\xi=0 \\
\tau=-e^{-t} k_{1}+k_{2} \\
\phi=-e^{-t} k_{1} u .
\end{gathered}
$$

Hence, the symmetry algebra of Equation (3) is two dimensional and is spanned by

$$
X_{1}=-e^{-t} \frac{\partial}{\partial t}-e^{-t} u \frac{\partial}{\partial u} \quad \text { and } \quad X_{2}=\frac{\partial}{\partial t} .
$$

The reduction via $X_{2}$ will lead to t-invariant solutions which are not of interest, so we reduce Equation (3) by exploiting the symmetry $X_{1}$. Solving the characteristic system for $X_{1} I=0$ gives the similarity variables for $X_{1}$ as

$$
z(x, t)=x \quad \text { and } \quad V(z)=e^{-t} u .
$$

Using these similarity variables reduces Equation (3) to the second order ODE

$$
z V \frac{d^{2} V}{d z^{2}}+z\left(\frac{d V}{d z}\right)^{2}+V \frac{d V}{d z}-z V^{2}=0 .
$$


Further, it is easy to see that Equation (5) admits the symmetry $X=V \frac{\partial}{\partial V}$. The similarity variables for $X=V \frac{\partial}{\partial V}$ are

$$
s=z \quad \text { and } \quad w(s)=\frac{V^{\prime}}{V},
$$

which reduce it to the Riccati equation

$$
\frac{d w}{d s}=-2 w^{2}-\frac{1}{s} w+1 .
$$

In order to solve the Riccati equation (7) we substitute

$$
w(s)=\frac{y^{\prime}(s)}{2 y(s)}
$$

to get

$$
s^{2} \frac{d^{2} y}{d s^{2}}+s \frac{d y}{d s}-2 s^{2} y=0 .
$$

This is modified Bessel equation of order $\nu=0$ whose general solution is

$$
y(s)=a_{1} I_{0}(\sqrt{2} s)+a_{2} K_{0}(\sqrt{2} s)
$$

where $I_{0}(x)$ and $K_{0}(x)$, respectively, denote modified Bessel functions of first and second kind of order zero.

Writing $\frac{a_{1}}{a_{2}}=C_{1}$ and using Equation (8) we see that the general solution of Riccati Equation (7) is given by

$$
w(s)=\frac{1}{2}\left\{\sqrt{2} \frac{C_{1} I_{1}(\sqrt{2} s)-K_{1}(\sqrt{2} s)}{C_{1} I_{0}(\sqrt{2} s)+K_{0}(\sqrt{2} s)}\right\} .
$$

Finally using the substitutions given by Equations (4) and (6) we obtain an exact solution of the Fisher Equation (3) in cylindrical coordinates, given by

$$
u(x, t)=C_{2} e^{t} \sqrt{C_{1} I_{0}(\sqrt{2} x)+K_{0}(\sqrt{2} x)} .
$$

Because the equation under investigation was studied over cylindrical domains, as expected the solution involves Bessel functions. The asymptotic behavior of the solution can be understood from the well known asymptotic behavior of the modified Bessel functions [8] which is

$$
I_{0}(x) \sim \frac{e^{x}}{\sqrt{2 \pi x}}, \quad K_{0}(x) \sim \frac{e^{-x}}{\sqrt{\frac{2}{\pi} x}}
$$

for large $x$.

\section{Acknowledgement}

The authors thank King Fahd University of Petroleum and Minerals (KFUPM), Saudi Arabia for the support and research facilities provided to complete this work. 


\section{References}

[1] G. Bluman and G. D. Cole, The general similarity solution of the heat equation, J. Math. Mech. 18, 1025 (1969)

[2] G. Bluman and S. Kumai, Symmetries and Differential Equaions, SpringerVerlag, New York, 1989.

[3] P. K. Brazhnik and J. J. Tyson, On travelling wave solutions of Fisher's equation in two spatial dimensions, SIAM J. Appl. Math. 60, 371 (1999)

[4] B. J. Cantwell, Introduction to Symmetry Analysis, Cambridge University Press, 2002.

[5] P. Kaliappan, An exact solution for travelling waves of $u_{t}=D u_{x x}+u-u^{k}$, Physica D 19,368 (1984).

[6] J. D. Murray, Mathematical Biology, Springer-Verlag, 1991.

[7] M. N. Ozisik, Heat conduction, John Wiley, 1993.

[8] G. N. Watson, A treatise on te theory of Bessel functions, second edition, Macmillan Publishing Co., Inc., New York, 1945. 